\title{
An Enhanced LID Routing Security Scheme for Mobile Ad-Hoc Networks
}

\author{
${ }^{1}$ Dr. M. Mohamed Musthafa and ${ }^{2}$ Dr. S.Karthik \\ 1 Professor \& Vice Principal, Dept. of CSE, Al-Ameen Engineering College, Erode, Tamilnadu, India \\ musthafain@gmai.com \\ 2Professor \& Dean, Dept. of CSE, SNS College of Technology, Coimbatore, Tamilnadu, India
}

\begin{abstract}
In this work we present novel security architecture for MANETs that merges the clustering and the threshold key management techniques. The proposed distributed authentication architecture reacts with the frequently changing topology of the network and enhances the process of assigning the node's public key. In the proposed architecture, the overall network is divided into clusters where the cluster heads $(\mathrm{CH})$ are connected by virtual networks and share the private key of the Central Authority (CA) using Lagrange interpolation. Experimental results show that the proposed architecture reaches to almost $95.5 \%$ of all nodes within an ad-hoc network that are able to communicate securely, 9 times faster than other architectures, to attain the same results. Moreover, the solution is fully decentralized to operate in a large-scale mobile network. We also proposing a special security routing architecture called Local Intrusion Detection (LID) to detect Black Hole Attack (BHA) over Ad hoc On Demand Distance Vector (AODV) MANET routing protocol. In LID security routing mechanism, the intrusion detection is performed locally using the previous node of the attacker node instead of performing the intrusion detection via the source node as in Source Intrusion Detection (SID) security routing mechanism. By performing LID security routing mechanism, the security mechanism overhead would be decreased.
\end{abstract}

\section{KEYWORDS}

Mobile Ad-hoc network, Ad hoc On Demand Distance Vector, Local Intrusion Detection, Source Intrusion Detection, Certificate Authority (CA) and Warrant node.

\section{INTRODUCTION}

The powerful topology of Mobile Ad Hoc Networks (MANETs), provides significant difficulties to key control and authentication techniques. Five specifications can be determined for any certificate-based authentication plan to be considered protected and efficient, with regards to the verification functions in a mobile ad hoc system. These are:

1. Allocated authentication: In an ad-hoc system (Ahmed.A, 2005; Jun-Zhao Sun, 2001; Yih-Chun Hu, Adrian Perrig, 2004) due to issues such as regular link problems, node flexibility, and restricted wireless method, it is generally not possible to include a set central CA in the system. Further, in networks demanding great protection, such a hosting server could become a anchorman of failing. Thus, one of the primary specifications of a certificate-based procedure is to spread the verification amongst a set of nodes in the system.

2. Source awareness: Since the nodes in an ad hoc system generally run on battery power with great energy intake and low storage potential, the verification techniques must be resource-aware. That indicates the time and space complexness of the actual techniques must be acceptably low. In this regard, symmetric-key-based cryptographic techniques are more suitable, ascompared to community key techniques, since symmetrical cryptography in general have less resource intake. However, the problem of circulating the symmetrical important factors stops their practical implementation in ad hoc networks. This is a compromise that must be handled at the program level. Since the certificate-based verification uses community key systems, which is resource intense, the method itself that is depending on accreditations must be efficient both in terms of storage and energy.

3. Efficient qualifications control mechanism: The submission of community important factors and control of accreditations have been analyzed substantially in the situation of wired networks. However, in implementing these techniques to MANETs, handling the accreditations (creation, cancellation and renewal) is a challenging problem. Most of the current systems lack a solid qualifications cancellation plan.

4. Heterogeneous certification: As in the situation of wired networks, the validating regulators might be heterogeneous even in ad hoc networks. This implies that two or more nodes that belong to different "domains" may try to verify each other. In such a situation, there must be some kind of believe in relationship or structure among the CAs. In wired networks,this is achieved through qualifications chaining.

5. Robust pre-authentication mechanism: By preauthentication procedure we mean the procedure of developing necessary believe in between nodes before the actual qualifications development and submission. Though this is not a part of the qualifications verification procedure itself, it is important in MANETs. It is compulsory that nodes have prior believe in between each other (by exchange of community important factors, for example). Without this established, the later common verification and restoration of accreditations would not be possible. Therefore, it is not possible to apply a central, reliable enterprise for handling community important factors of the members as conducted in regional community networks or the Internet. Instead, a distributed solution must be found. In this document, we recommend and assess a

$4549 \mid \mathrm{P}$ a g e 
novel structure for protected interaction in MANETs. Our approach separates the system into groups and utilizes a decentralized qualifications power. Decentralization is obtained by using limit cryptography and a system secret that is shipped over a number of nodes. While this essence has been suggested earlier, its program on a grouped system is the unique of our perform.

Several initiatives in (Al-Shurman M., and S. Yoo, 2004; Deng H. et al, 2002; Wang D. et al, 2008) have been made to improve different redirecting techniques with protection techniques and features. Including more protection techniques and features on the redirecting criteria indicates adding more handling expense and causing more system performance deterioration. LID protection redirecting procedure is an enhancement of SID protection redirecting procedure over AODV MANET redirecting method For the best of our knowledge, there is no one had enhanced SID protection redirecting procedure in (Al-Shurman M., and S. Yoo, 2004). Both SID and LID protection redirecting systems identify BHA over MANET to prevent the risk of fabricating AODV redirecting information by BHA. However, LID protection redirecting procedure makes AODV redirecting method efficient in the both protection and system performance dimensions. The main enhancement in LID protection redirecting procedure over SID method is using regional attack recognition systems that is conducted on the past node of the advanced node/attacker node on the path, according to AODV redirecting method, instead of over filling the system with extra path to perform the attack recognition by the source node itself as in SID protection redirecting (Al-Shurman M., and S. Yoo, 2004). By doing regional attack recognition, as in our LID protection redirecting procedure, the protection systems expense would be reduced. This document is organized as the following: Section 2 describes the related work. Section 3 describes the proposed security architecture. Section 4 provides the LID security routing mechanism. Area 5 provides the simulation and result analysis, and finally conclusion.

\section{RELATED WORK}

A procedure to sustain CRLs (certificate cancellation lists) centered on customer profile and position platforms was suggested in (Claude Crepeau and Carlton R. Davis, 2003). Their plan manages the issue of harmful nodes revoking the accreditations of believe in deserving nodes. However, the supposition that each node knows the depend of the variety of nodes in the system at any immediate might not be possible to apply in a genuine situation. Many scientists have analyzed the key control issue in ad hoc systems. For example, (Kong J., P. et al, 2001; Luo H., and S. Lu., 2000) explain a completely allocated PKI remedy in which all nodes discuss the qualifications power deciding upon key according to the $(K, N)$ limit plan where $\mathrm{N}$ is the depend of hosting server nodes and $\mathrm{K}$ is the lowest variety needed to recalculate the key key. In this plan, the writers suggested a allocated qualifications depending on limit cryptography and distributed tricks. The primary objective of a limit key discussing technique is to discuss a key key $S$ among an randomly huge group using a key polynomial $f(x)$ (Luo H., and S. Lu., 2000). If the level of $f(x)$ is (k-1), then any $k$ associates of the group can restore the key key, while less than $\mathrm{k}$ associates expose no details of the key. To discuss the qualifications deciding upon key $\mathrm{S}$ between $\mathrm{N}$ customers, a polynomial operate of level $\mathrm{K}-1<\mathrm{N}$ is regarded as follows, in system (1):

$$
F(x)=(S+a 1 x 1+a 2 \times 2+\ldots+a k-1 \times k-1) \bmod p
$$

Where $F(0)=S$ (the key key) and $p$ is a huge primary variety and a1, a2 .., and ak-1are randomly selected from Z/PZ. Then each customer of identification idi is offered with its partially key $\mathrm{Si}=\mathrm{f}$ (idi). Their stocks offer $\mathrm{k}$ unique factors $(\mathrm{x}, \mathrm{y})$ $=(i, \mathrm{Si})$ enabling calculations of the coefficients $a j, \quad I \leq j \leq k-1$ of $f(x)$ by Lagrange interpolation system (2): Lagrange interpolation

$$
F(X)=\sum_{i=1}^{k} Y i \prod_{1 \leq j \leq k, j \neq i} \frac{X-X j}{X i-X j}
$$

Since $f(0)=a 0=S$, the shared secret can be expressed as equation (3):

$$
S=\sum_{i=1}^{k} C i Y i \quad \text { where } C i=\prod_{1<j \leq t} \frac{X j}{X j-X i}
$$

The key $\mathrm{S}$ is then measured from $\mathrm{S}=\mathrm{F}(0)$. Thus, a set of $\mathrm{K}$ working together customers can restore the qualifications deciding upon key S. In this remedy, solutions offered by the CA (Central Authority) such as qualifications restoration or cancellation, except the qualifications assistance (certificate delivery) which is achieved by the CA, are dedicated to all nodes in the system. A overview of this strategy is proven in Determine 1. Actually, a function demanding the CA's personal key cannot be done without the contribution of $\mathrm{K}$ or more working together nodes. So, this remedy represents that every node has at the least $\mathrm{K}$ others who live nearby and that the latter must acquire an preliminary qualifications from the CA before becoming a member of the system. One of the certificate-based verification techniques recommended by (Capkun et al. 2003) is development qualifications charts (Yi, S., and Kravets, 2002 \& 2004; Capkun $\mathrm{S}$. et al, 2003). The recommended strategy is just like Fairly Excellent Comfort (PGP), public-key cryptography application which is easily available over the Online (Zimmermnn, P., 1995).

This application has become the de facto conventional for the security of email and information. PGP and its writer John p Zimmermann are the focus of nationwide and worldwide controversy concerning this new, highly effective "envelope"

$4550 \mid P$ a g e 
that allows people the same comfort in emails as experienced by government authorities and large organizations, apart from the fact that in PGP a main certification hosting server is used. They determine a certification chart as a instructed chart $G(V, E)$ where $V$ and $E$ are the set of vertices and the set of sides, respectively. The vertices of the certification chart signify community important factors, and the sides signify accreditations. As proven in Determine 2, a instructed advantage from vertex $\mathrm{Ku}$ to $\mathrm{Kv}$ symbolizes the certification from $u$ to $v$ by u's deciding upon v's community key $\mathrm{Kv}$ with its own personal key. Thus, $u$ became the CA for $v$. The chart $G$ contains only the legitimate accreditations of the whole system. The achievements of this strategy very much relies on the development of the regional certification databases and on you will of the certification charts. The vertices of a certification chart signify public-keys of the customers and the sides signify public-key accreditations from the customers. The writers examine several database development methods and research their performance. The suggested methods take into consideration you will of the certification charts in a feeling that the choice of the accreditations that are saved by each cellular node relies on the connection of the node and its others who live nearby in the certification chart.

More accurately, each node shops in its regional database several instructed and mutually disjoint routes of accreditations. Each direction starts at the node itself, and the accreditations are included to the direction such that a new certification is selected among the accreditations linked with the last node on the direction (initially the node that shops the certificates), so that the new certification results in the node that has the biggest number of accreditations linked with it (i.e., the biggest vertex degree). The writers call this criterion the highest possible Level Algorithm, as the regional database development requirements is the level of the vertices in a certification chart.

The main concept of this strategy is described in Fig. 1.and reveals the regional certification databases of customers $u$ and $v$ and the stores of accreditations that $u$ uses to verify the community key Kv of $v$. The community important factors of the customers are showed by certification chart vertices, while the chart sides signify public-key accreditations from the entrepreneurs of the community important factors.

\section{PROPOSED SECURITY ARCHITECTURE}

The common framework and function of the proposed work namely; LID, secured routing architecture are described as follows:

Bootstrapping: We partition the system into clusters; each of them has one group Cluster Head $(\mathrm{CH})$ that is accountable for developing and planning the group. Entrance nodes are accountable for handling interaction between nearby groups. The $\mathrm{CH}$ nodes are accountable for delivering $\mathrm{CH}$ beacons in their groups containing management details for the group associates, e.g. details of nodes and GWs in the group. GWs regularly transfer GW beacons to notify their specific groups about nearby groups. The reasons for our security idea are the use of community key cryptography for guaranteeing verification, reliability and privacy. Every node in the system maintains a self-produced key couple, whereas the community important factors are allocated using accreditations from a allocated CA that established by the group go nodes. For giving accreditations certain discuss of these $\mathrm{CH}$ nodes should definitely take position.

This idea has two advantages: first of all, accessibility is improved, because accreditations can be released even if some qualifications nodes are not available for the new nodes since the $\mathrm{CH}$ appears like them for the challenging node if it is reliable to its Guarantee nodes, and secondly, the protection framework becomes more immune to criminals since any new node cannot need straight the deciding upon of its community unless it is reliable from a known variety of the guaranteed nodes reliable from the $\mathrm{CH}$. The $\mathrm{CHs}$ type a sensible system known as $\mathrm{CH}$ system. The personal key of the $\mathrm{CA}$ is allocated over the $\mathrm{CHs}$, every $\mathrm{CH}$ maintains a discuss. There is also a symmetrical key for every group known to the group associates produced by its group go. It is useful for group inner visitors, and to cover up details such as the resource and location details from the eavesdroppers not from the group.

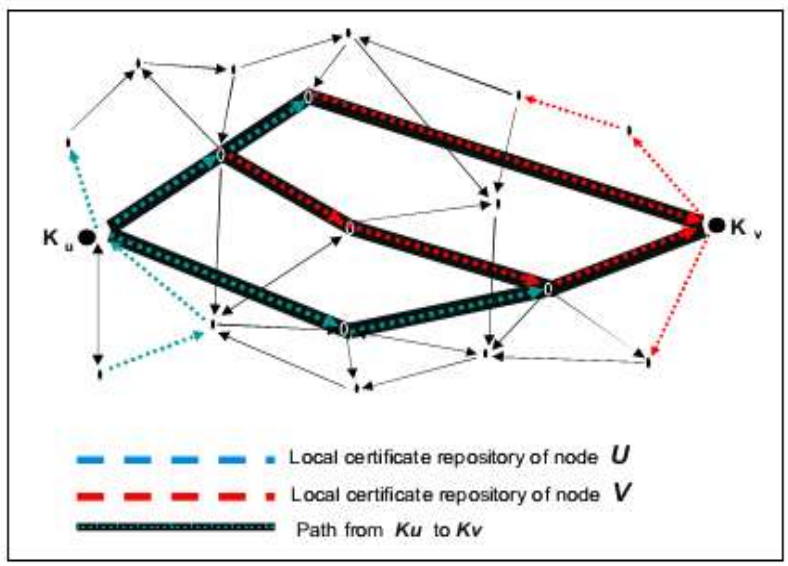

Fig. 1: Self-structured public key management

4551 I $P$ a g e 


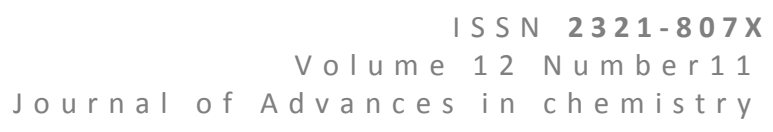

Key Management: The system key, which is distributed amongst the $\mathrm{CHs}$ of an ad hoc system, is designed using practical key discussing (Fokine K., 2002 ; Wu, B. et al, 2005) according to the Electronic Trademark Plan. The structure of the $\mathrm{CH}$ system changes dynamically as $\mathrm{CHs}$ be a part of or keeps the system. The key stocks also have to be restored consistently because the variety of stocks needs to be tailored to the variety of CHs. Apart from that, it has to create sure that the key stocks are restored after a certain time interval to create it difficult for a shifting enemy to bargain a variety of $(\mathrm{k}) \mathrm{CH}$ eventually. In the suggested strategy, we always merge the becoming a member of or making of $\mathrm{CHs}$ with a key discuss restoration and only routine extra restoration if the $\mathrm{CH}$ system continues to be the same for a while.

The community key of the $\mathrm{CH}$ system should be known to all nodes in the ad hoc system. It is spread via the $\mathrm{CH}$ beacons showed consistently in every group. Besides the community system key, a $\mathrm{CH}$ shining example also contains the CHs own community key, a record of nodes of the present group such as their position, and a record of gateways joins nearby groups. New nodes are required first to obtain a certain variety of assurance accreditations from other system nodes (warranting nodes that will be described in the sign-up phase) that are others who live nearby to the new node, where individual get in touch with between individual customers is possible and allows for verification.

\section{LID SEURITY ROUTING MECHANISM}

In purchase to minimize the disadvantages in SID security routing procedure suggested in (Al-Shurman M., and S. Yoo, 2004). We recommend new procedure known as Regional Attack Recognition (LID) security routing procedure (Fig. 2) to allow the detection of the enemy to be locally; which indicates that when the alleged advanced node (node N5) unicast the RREP towards the resource node (node N1) the past node (node N4) to the advanced node functions the procedure of detection and not the resource node. First, the past node buffers the RREP bundle. Second, it uses a new path to the next node (node N6) and delivers FRREQ bundle toit. When the past node gets the FRREP bundle from the next hop node, it ingredients the details from the FRREP bundle and acts according to following rules:If the next node (N6) has a path to advanced node (N5) and location node (N7), the past hop node eliminate the FRREP, then unicast the RREP to the resource node.

If the next hop (N6) has no path to the location node (N7) or the advanced node (N5) or both of them (N5 and N7), the past node (N4) discards the buffered RREP and the FRREP as well, simultaneously programming the alert concept to declare there is no properly secured enough path available to the location node (N7).

The last situation contains another situation such as; the situation in which the past hop node does not get any FRREP from the next hop node. So, here the resource node will find out a new path to the location. This will reduce both routing expense packages and end-to-end wait, and will improve the system throughput simultaneously.

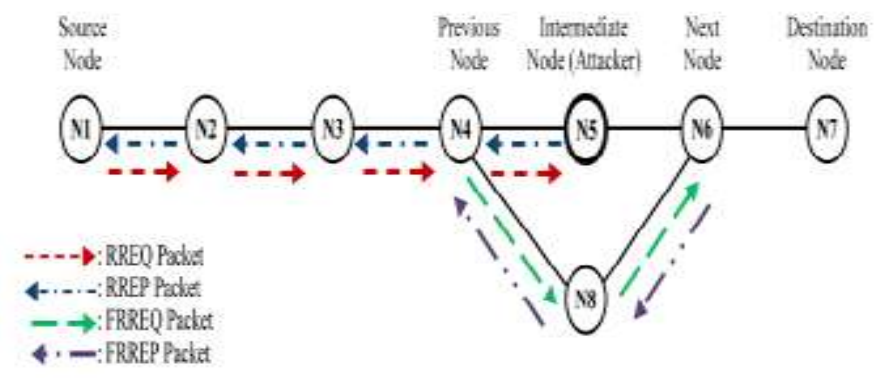

Fig. 2: Proposed LID Security Routing Mechanism

The pseudo code LID secure mechanism is show in Fig. 3. The Fig. 4, Fig. 5, compare between the program throughput, frequent end-to-end hang on, and direction-finding cost respectively in both LID and SID security direction-finding systems while different the number of nodes. It is clear from the numbers that LID security direction-finding process outperforms SID security direction-finding process. This is because LID security direction-finding process uses local strike identification rather than SID security direction-finding process that uses source strike identification. LID security direction-finding reduces direction-finding information cost which results in to less populated program and less utilized information usage

4552 | P a g e 


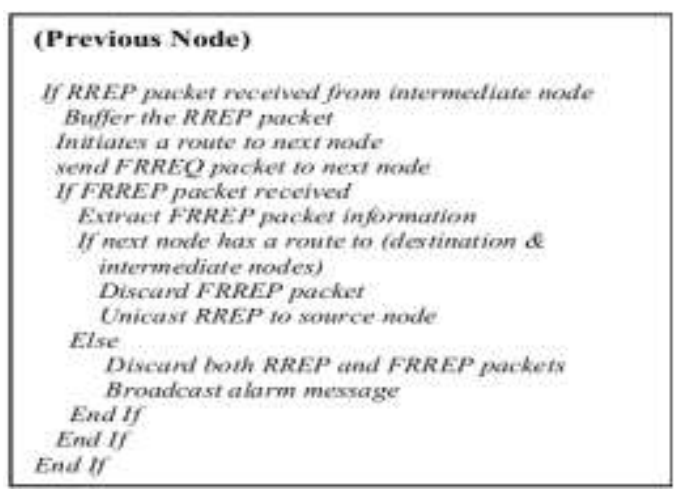

\begin{tabular}{|l|}
\hline (Source Node) \\
Broadcast RREQ poncket \\
UfREPP pochet necerved \\
send data packets \\
Else \\
Reinitiaties a new RREQ packet \\
End If
\end{tabular}

Fig. 3: Pseudo code for LID Security Mechanism

which reduces the losing information offers and to have an increase in program throughput and loss of both end-to-end hang on and direction-finding cost. According to this research, the improvement rate of throughput, frequent, end-to-end hang on, and direction-finding cost obtained by LID security direction-finding are $1.2 \%, 10.3 \%$, and $3.4 \%$ respectively.

\section{PERFORMANCE EVALUATION}

To imitate the performance of LID security redirecting procedure, we use GloMoSim 2.03 system simulation (Lokesh B., et al, 1999). GloMoSim is system method simulation software that models wireless and wired system techniques. Our select for GloMoSim based on its ability to run under windows environment and it uses a levels approach which is currently used by most system techniques. Table 1 shows simulation factors that are used along all of our models tests.

Table1: Simulation Parameters

\begin{tabular}{|l|l|}
\hline Parameter & Value \\
\hline Routing & AODV \\
\hline Connection & 10 CBR \\
\hline Node Placement & Random \\
\hline Mobility speed & $0-10 \mathrm{~ms}$ \\
\hline MAC & 802.11 \\
\hline Data Packet Size & 512 bytes \\
\hline
\end{tabular}

We believe that every node has a set transmitting variety, $r=120 \mathrm{~m}$. Two nodes are others who live nearby if the range between them drops within the transmitting variety. Wait per hop is $150 \mathrm{~ms}$. CHs transmitted their beacons over 2 trips every 20s. To obtain complete account a visitor needs to obtain the accreditations from the lowest variety of guarantee needed at its group to get its identification certification. The lifetime for the certification is selected arbitrarily between 150 s to 200 s.Warrant responded to to guarantee demands with beneficial rate of $50 \%$. Each statistic composed 40 models each of which operates in 420s. Usually, any node can be authenticated, in the best situation, after it creates a variety of trips is equal to $\left(2{ }^{*}\right.$ min guarantee nodes needed to be reliable from the $\left.\mathrm{CH}\right)+2$. The simulator was applied using the simulator flip NS2, published with C++ terminology (Fall.K, Vardhann.k, 2005).

Availability is the rate between the nodes that were provided the verification to the count of nodes that were looking for it. In other terms, the provision is a evaluate of the amount of all nodes within the ad hoc systems that are able to connect safely. Determine 4 reveals the outcomes of the suggested framework with $\mathrm{CH}$ shining example durations of $10 \mathrm{~s}$ and 30 s. From the figure you can differentiate two phases: the installation stage (approximately the first 50s), during which the framework is being recognized and roughly $64 \%$ of the nodes are able to connect safely. 

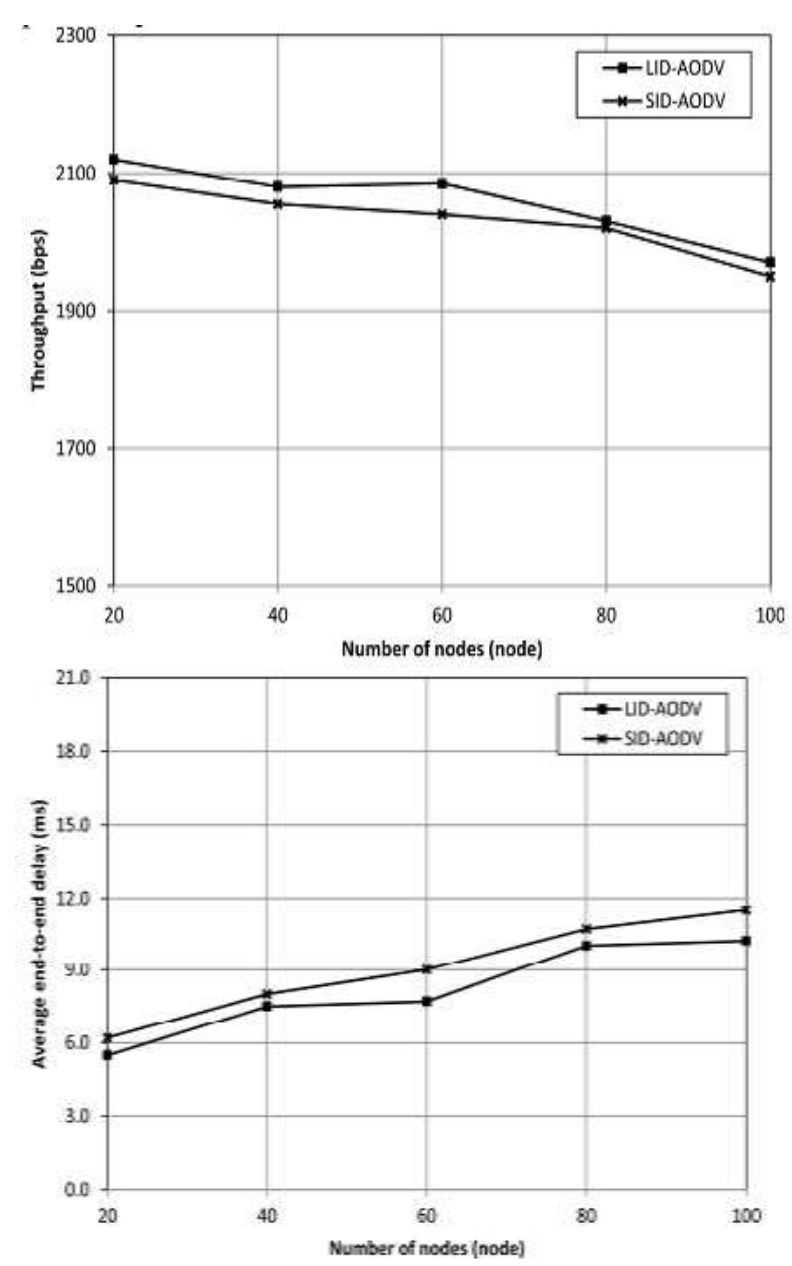

Fig. 4: and Fig. 5: compare between the network throughput, average end-to-end delay

The second stage comes next (after about 60s) when the group topology and the protection facilities are well recognized allowing about $95.5 \%$ of the cellular nodes to connect safely. Simulated outcomes display that the suggested framework accomplishes better accessibility in a smaller time as opposed to self-organizing public-key control program discovered in (Capkun S. et al, 2003), see Determine 4. From the documented outcomes it can be observed that the suggested framework obtained almost $95.5 \%$ of all nodes within the ad hoc systems that are able to connect safely in a $115 \mathrm{~s}$. A identical stage was obtained by(Capkun S. et al, 2003), but after 1000s for the same simulator factors.

Register Time: A cellular node's logon time is calculated as enough interval of time between getting a $\mathrm{CH}$ shining example and obtaining a complete account in the group. Within this log-on time, Fig. 6 and Fig. 7 show the cellular node has to find the lowest number of the assurance nodes required as each group plan gathers three stocks of the identitybased key certification.

Lastly, it brings together the stocks and demands the shaped group key from the group go. Preferably, the logon process should be short to assurance fast acceptance of the cellular nodes to the ad hoc system.A simulated run for the log-on interval of time in Fig. 8 was conducted as follows: first, nodes are allocated arbitrarily selected types (cluster go, entrance, or visitor node). Cluster leads are sent their beacons every $20 \mathrm{~s}$ via 2 trips. In the second step, all nodes started to move arbitrarily and the visitor nodes tried to acquire a complete account. Determine 5 reveals the common result of a run. Determine 6 reveals that at the starting about $20 \%$ of the nodes were authenticated. Next, $8 \%$ more nodes were efficiently authenticated and so on. The regular logon here we are at the new nodes was about $23.7 \mathrm{~s}$. Nodes accessibility achieved $39 \%$ in the first $20 \mathrm{~s}$. 


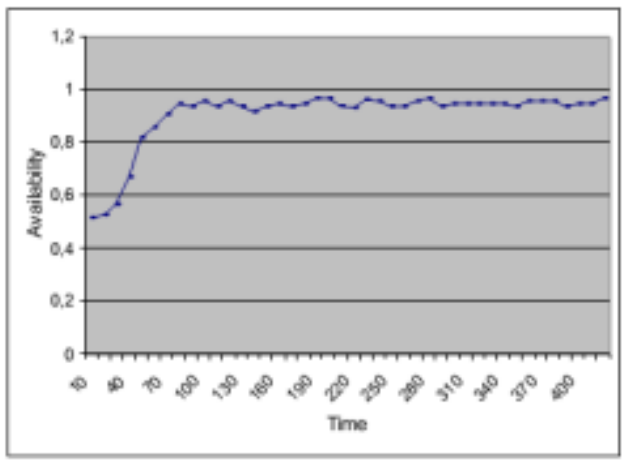

Fig. 6: Availability Performances of new node

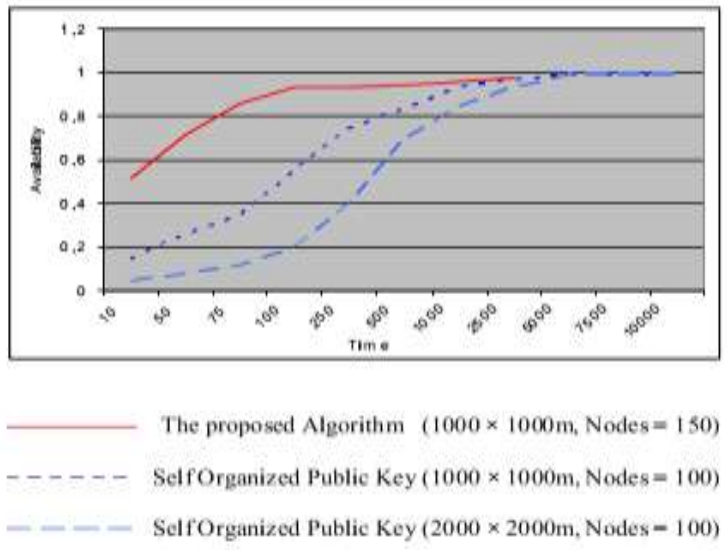

Fig. 7: Accessibility to Nodes for the Suggested \& Current Architectures

Unsuccessful nodes that fail to register can try again in the authentication process after 50 s. The log-on procedure of a guest node can fail for one of the following two reasons: first, the guest is not able to collect enough warranty certificates within the predefined period of time;second, after having received a $\mathrm{CH}$ beacon, the guest cannot communicate with the $\mathrm{CH}$ because of the dynamic nature of the network topology.

Packet Overhead: Security protocols always cause additional overhead. The proposed security architecture defines different types ofmobile nodes(cluster head, gateways nodes, full members).

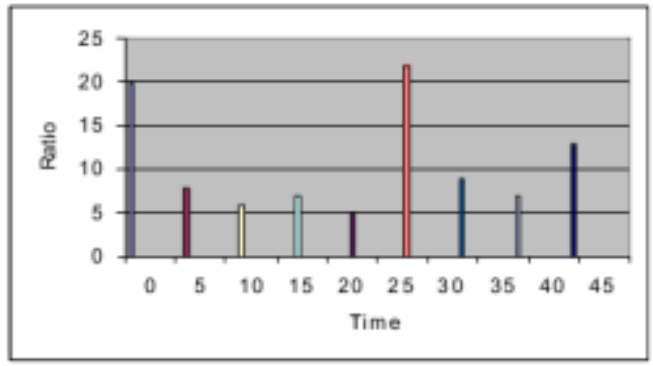

Fig. 8: Logon time for new nodes 


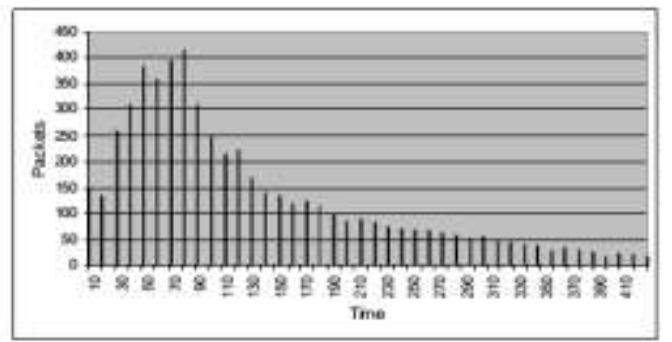

Fig. 9: Packets Overhead

A trace for the overall network traffic was drawn. Results reveal that in the setup phase, the overhead is relatively high due to establishing the security infrastructure. This overhead drops down to less than 40 backets/s, as illustrated in Fig. 9.

\section{CONCLUSION}

The provided work established a remedy to protection support in wireless cellular systems. The method is self structured, scalable and versatile. It looks for to quickly increase the service accessibility in each system area which is crucial for cellular users. The remedy was fully decentralized to function in a large-scale cellular system. Although the results achieved in this document were achieved with the other scientists, the suggested structure. This document also suggested LID protection redirecting procedure over AODV MANET redirecting method. LID protection redirecting functions its attack recognition procedure regionally in the previous node of the advanced node in comparison with SID protection redirecting procedure, which functions its attack recognition procedure by the source node. End-to-end wait, redirecting expense, and throughput of SID andLID protection redirecting systems were compared by different the number of nodes.

\section{REFERENCES}

1. Ahmed.A, 2005."Wireless and mobile data networks", Wiley.

2. Al-Shurman M., and S. Yoo, 2004. "Black hole attack in wireless ad hoc networks," in ACM 42nd Southeast Conference (ACMSE'04), pp. 96-97.

3. Capkun S., L. Buttyanet J-P Hubaux, 2003. "Self-Organized Public- Key Management for Mobile Ad Hoc Networks", ACM International Workshop on Wireless Security.

4. Claude Crepeau and Carlton R. Davis, 2003."A Certificate Revocation Scheme for Wireless Ad hoc Networks", Proceedings of the 1st ACM workshop on Security of ad hoc and sensor networks, Fairfax, Virginia.

5. Deng H., W. Li and D. Agrawal, 2002."Routing security in ad hoc networks,"IEEE Communications Magazine, vol. 40, no. 10 , pp. $70-75$.

6. Fall.K, Vardhann.k, 2005." The ns Manual (formerly ns Notes and Documentation)", The VINT Project a Collaboration between researchers at UC Berkeley, LBL, USC/ISI, and Xerox PARC.

7. Fokine K., 2002. "Key Management in Ad-Hoc Networks", Mastersthesis, University of Linkopings, Sweden.

8. Jun-Zhao Sun, 2001. "An essential technology for pervasive computing", National Technology Agency of Finland, 2001

9. Kong J., P. Zerfos, H. Luo, S. Lu and L. Zhang, 2001."Providing robust and ubiquitous security support for mobile ad-hoc networks", ICNP, pp. 251-260.

10. Lokesh B., T. Mineo, A. Rajat, T. Ken, B. Rajive and G. Mario, 1999."GloMoSim: A Scalable Network Simulation Environment," Technical Report 990027, University of California.

11. Luo H., and S. Lu., 2000. "Ubiquitous and Robust Authentication for Ad-Hoc Wireless Networks", Technical reports TR-200030,Dept of UCLA.

12. Wang D., M. Hu and H. Zhi, 2008."A survey of Secure Routing in Ad Hoc Networks," IEEE 9th International Conference on Web Age Information Management, pp. 482-486.

13. Wu, B., Mohamed llyas and Jie Wu, 2005."Secure and efficient key management in mobile ad-hoc networks", journal of Network and Computer Applications, Elsevier.

14. Yi, S., and Kravets, 2002.R."Key Management for Heterogeneous Ad Hoc Wireless Networks", Network Protocols, 2002.Proceedings.10th IEEE International Conference, pp. 202- 203.

15. Yi, S., and Kravets, R., 2004. "MOCA: Mobile Certificate Authority for Wireless Ad-Hoc Networks", Report No. UIUCDCS-R-2004.

16. Yih-Chun Hu, Adrian Perrig, 2004. "A survey of secure wireless ad hoc routing" published by the IEEE Computer Socity.

17. Zerfos. P, Kong.J, 2004. "Providing Robust and Ubiquitous security support for mobile Ad-Hoc Networks", CS department university o California At Los Angeles.

18. Zimmermnn, P., 1995. "The Official PGP usrs guide", MIT press. 